\title{
Über die pharmakologische Wirkung von Clitocybe acromelalga, Ichimura.
}

\author{
Von \\ Osamu Miura. \\ (三浦修) \\ (Aus dem Pharmakologischen Institut [Direktor: Prof. Dr. \\ S. Yagi] und der Hautklinik [Direktor: \\ Prof. Dr. M.Ota] zu Sendai.)
}

Clitocybe acromelalga, Ichimura ist ein zu den Agaricaceen gehörender Pilz, der in Japan stellenweise im Herbst vorkommt und nur selten gegessen wird. Einige Menschen können ihn zwar ohne Schaden geniessen, bei anderen ruft er aber manchmal mehr oder weniger schwere Vergiftungen hervor. Dabei ist man sich im unklaren darüber, ob es sich in den erstgenannten Fällen um eine Toleranz handelt oder nicht. Unter den Vergiftungserscheinungen finden sich weder Hirnsymptome noch Magen- und Darmstörungen, die bei verschiedenen anderen Pilzvergiftungen innerhalb eines Tages aufzutreten pflegen. Erst 1 bis 8 Tage nach dem Genuss machen sich hauptsächlich an den Vorderarmen und Händen, sowie an den Unterschenkeln und Füssen und selten auch im Gesicht Rötungen und Anschwellungen begleitet von Hitzegefühl und Schmerz bemerkbar, die an Erythromelalgie erinnern. Der Schmerz ist anhaltend und in der Regel sehr heftig, so dass die Kranken gar nicht einzuschlafen rermögen. Dieses Symptom lässt sich durch Verabreichung von Morphin kaum beseitigen, aber durch Eintauchen des erkrankten Körperteils in kaltes Wasser wird der Schmerz einigermassen gelindert. Aus diesem Grunde suchen die Kranken, um die Beschwerden zu mildern, die Glieder fortwährend in ein Wasserbad zu halten. Die Dauer der Symptome ist recht verschieden, sie halten einige Tage bis Wochen, ja sogar selten bis zu 70 Tagen an. Bei langem Verlauf werden die erkrankten Teile wahrscheinlich wegen des dauernden Eintauchens in Wasser von Mazeration und Nekrose befallen. Die letz- 
tere scheint aber nicht durch die Wasserbehandlung allein bedingt zu sein, da Vergiftungsfälle berichtet werden, bei denen trotz Nichtanwendens dieser Behandlung Gangräne auftreten. Schwere Fälle enden nicht selten letal.

Eine pharmakologische Untersuchung dieses Pilzes wurde zuerst von Inok $0^{1)}$ angestellt, der in seinem alkoholischen Extrakt ein Alkaloid nachweisen konnte, das nach seiner Wirkung auf Frösche mit dem Cholin identisch ist. Diesen Bestandteil machte der Autor aber für die Vergiftung des Menschen nicht verantwortlich, da sich bei Tierversuchen nur eine schwache Giftigkeit dieser Substanz ergab. Später teilte Iwak a wa $a^{2)}$ mit, dass der Pilz 2 Krampfgifte enthält, deren nähere pharmakologische Eigenschaften bisher aber noch nicht untersucht worden sind. Ausserdem sagte mir Dr. Sasaki, der Assistent in der Klinik Prof. Yamada's in Kanazawa, dass er bei japanischen Affen durch die Verfütterung dieses Pilzes überhaupt keine Vergiftungserscheinungen erzielen konnte. Trotz der Bemühungen dieser Autoren bleibt die pharmakologische Wirkung des Pilzes also so gut wie ganz unaufgeklärt, was mir den Anlass zu der vorliegenden Untersuchung gab.

\section{Vorbereitung des Versuchsmaterials.}

Obgleich die Clitocybe acromelalga in gekochtem Zustande gegessen wird und so die Vergiftung verursacht, ist es noch nicht klar, ob ihr giftiger Bestandteil gegen längeres Kochen absolut beständjg ist. Weiter ist es nach der Tatsache, dass der gakochte Pilz nach mehrmaligem Waschen mit Wasser keine Vergiftung mehr herbeiführt, wahrscheinlich, dass sein giftiger Bestandteil wasserlöslich ist. Deshalb wurde zunächst der getrocknete, zerschnittene Pilz mit der 10 fachen Menge Wasser bedeckt und unter oftmaligem Umschütteln bei Zimmertemperatur 2 Tage lang stehengelassen. Dann wurle filtriert und man erhielt eine dunkelbraune, halbdurchsichtige Flüs-

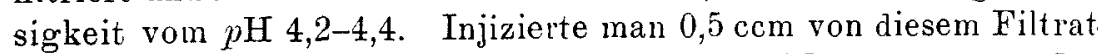
nach Neutralisieren mit Natriumkarbonat einer Maus von etwa $15 \mathrm{~g}$ Körpergewicht subkutan, so zeigte sie eigentümliche Bewegungen der Extremitäten, die das Vorhandensein von Hyperästhesie vermuten liessen, und Atembeschwerden, die schliesslich zum Tode führten. Die Extraktion des Pilzes wurde nun statt mit Wasser mit verschie-

1) Inok o, Tokyo Igakkai Zasshi, 1891, 5, 295.

2) I wak aw a, Jika-Zasshi, 1924, 99. 
denen organischen Lösungsmitteln auf diesclbe Weise ausgeführt und die erhaltenen Extrakte nach Befreiung von den Lösungsmitteln in Wasser aufgenommen, um ihre Wirkung an Mäusen zu prüfen. Dabei wurde festgestellt, dass der giftige Bestandteil in $95 \%$ igem Alkohol und Aeton nur wenig und in Petroleumäther, Äther, Chloroform sowie Benzol fast gar nicht löslich war. Diese Resultate veranlassten mich, die folgenden Versuche einstweilen mit dem wässerigen Extrakt vorzunehmen.

Der geschilderte $10 \%$ ige wässerige Extrakt zeigte in diesem $\mathrm{Zu}$ stand wie auch nach Neutralisierung durch den Zusatz von Natriumkarbonat beim Stehenlassen im Zimmer mehrere Tage lang keine Veränderung in seiner Toxizität, er verlor sie aber nach der Alkalisierung allmählich, bis diese nach mehreren Tagen nicht mehr nachgewiesen werden konnte. Der neutrale Extrakt erfuhr durch Destillation unter einem negativen Druck von $8-15 \mathrm{~mm} \mathrm{Hg}$ bei $40^{\circ} \mathrm{C}$ in bezug auf die Toxizität keine Veränderung. Deshalb wurde der wässerige Extrakt nach der Neutralisierung in einem Eisschrank aufbewahrt und jedesmal, wenn verlangt, herausgenommen. Nötigenfalls. wurde er unter den genannten Bedingungen auf beliebjge Konzentrationen eingedickt.

\section{Allgemeine Vergiftungserscheinungen.}

Die Vergiftungserscheinungen, die bei den Versuchen mit dem wässerigen Pilzextrakt an Fröschen (Rana jap.), Mäusen, weissen Ratten, Kaninchen und Meerschweinchen auftraten, waren je nach der Art des Tieres mehr oder weniger verschieden.

1) Frosch: Führte man in den abdominalen Lymphsack einer Rana japonica eine genügende Menge von dem 10\%igen Extrakt ein, so machten sich die Vergiftungserscheinungen nur langsam, und zwar erst etwa eine Stunde nach der Injektion bemerkbar. Die spontanen Bewegungen des Tieres wurden von da an immer seltener, um nach 4-6 Stunden völlig aufzuhören. Auch die reflektorischen Bewegungen wurden allmählich schwächer; versclswanden aber etwas später als die erstgenannten. Als die spontanen Bewegungen anfingen auffällig schwächer zu werden, wurde die Atmung langsamer und unregelmässig und setzte nach 2-3 Stunden endlich ganz aus. Das Her'z schlug dabei normal weiter, verfiel dann aber ganz allmählich und kam in etwa 12 Stunden zum Stillstand. Wenn man bei einem Frosch, bei dem die Motilität vollkommen gelähmt war, aber 
das Herz noch regelmässig und kräftig fortschlug, die Reizschwelle des $\mathrm{N}$. ischiadicus, bei welcher elektrische Schläge Zuckungen der betreffenden Muskeln hervorzurufen vermögen, prüfte, so sah man, dass sich der Schwellenwert kaum von dem eines normalen Frosches unterschied. Aus diesem Versuch geht hervor, dass der Pilzextrakt auf Gehirn und Rückenmark einwirkt und so das Verfallen und Verschwinden der spontanen, der reflektorischen und der Atembewegungen herbeiführt.

Die minimale Letaldosis des $10 \%$ igen Extraktes war pro $10 \mathrm{~g}$ Körpergewicht $0,4 \mathrm{ccm}$. Bei der Darreichung einer grösseren Dosis kamen die Vergiftungssymptome ungefähr ebenso spät zum Vorschein und verliefen annähernd ebenso schnell wie bei der minimalen Letaldosis. Spritzte man den Extrakt nicht subkutan, sondern in die Bauchvene ein, so betrug die minimale Letaldosis $0,04 \mathrm{ccm}$ pro $10 \mathrm{~g}$ Körpergewicht d.h. den zehnten Teil der vorigen. Dabei erfolgte der Stillstand des Herzens nach etwa 30 Minuten. Hiernach scheint der giftige Bestandteil des Extraktes vom subliutanen Gewebe sehr schwer resorbiert zu werden.

2) Maus: Wenn man einer Maus die minimale Letaldosis, d. h. $0,3 \mathrm{ccm}$ des Extraktes pro $10 \mathrm{~g}$ Körpergewicht subkutan einspritzte, so kauerte sie sich nach kurzer Zeit ganz ruhig nieder. Der Atem schien anfänglich schneller zu sein, fing aber dann an langsamex und anstrengend zu werden, und die Augäpfel treten nach und nach hervor. Nach 15 bis 50 Minuten wurde das Tier unruhig und lief im Käfig hastig umher. Währenddessen hielt es aber sehr oft an und leckte dabei die Brust und die Vorder- und Hinterbeine abwechselnd im schnellsten Tempo ab, indem es sich auf die Hinterbeine setzte, oder kratzte sich abwachselnd mit den Hinterbeinen an den Flanken, als ob es von den Flanken oder Füssen irgendetwas wegschaffen wollte. Der Wechsel von Laufen und Anhalten wurde mit der Zeit immer schneller, so dass die Maus gar keine Zeit mehr hatte, sich auszuruhen. Dann lief sie nur auf den Zehen, als ob sie möglichst vermeiden wollte, dass die Füsse den Boden berührten. Infolgedessen wurde das Laufen immer unsicherer und taumelnder, so dass sie später sehr häufig umfiel und schliesslich nicht mehr imstande war, sich aufrecht hinzusetzen. Dabei machte sie bald um den Kopf Kreisbewegungen, bald um die Körperachse Drehbewegungen, indem sie die Extremitäten schüttelte. Die Maus reagierte num nicht mehr auf Klopfen an die Gefässwand sowie auf Händeklatschen, während sie auf die leiseste Berührung der Extremitäten oder des Schwan- 
zes mit einem Stäbchen sogleich mit starkem Hüpfen antwortete. Indessen wurde die Atmung sehr langsam und mühsam, und stand schliesslich im Todeskrampf still. Bei sofortigem Öffnen der Brust sah man das Herz noch schlagen, es kam aber nach wenigen Minuten zum Stillstand. Die Lungen sahen stark hyperämisch aus.

Bei nicht letalen Dosen traten die oben beschriebenen Symptome in verschiedener Stärke ein, hielten lange Zeit an und verschwanden ganz allmählich. Bei der Hälfte der minimalen Letaldosis erholte sich die Maus z. B. erst nach 2 Tagen von den genannten Vergiftungser'scheinungen. In solchen Fällen sah man an der Injektionsstelle stets eine Induration, die nach 4-5 Tagen in eine trockne Nekrose überging. Auf keinen Fall war aber eine Rötung oder Anschwellung der Extremitäten bemerkbar, die bei der Vergiftung des Menschen charakteristische Symptome bilden.

Der Pilzextrakt bewirkt also bei der Maus neben einer Lokalreizung eine Verlangsamungsdyspnoe, die sich zum Atemstillstand entwickelt, und ein eigentümliches Betragen, das wahrscheinlich von einer Hyperästhesie herrührt. Das letzte mag vielleicht mit der bei der Vergiftung von Menschen vorkommenden sensiblen Störung im Wesen identisch sein.

3) Ratte: Die subkutane Verabreichung des Extraktes führte bei Ratten eine Verlangsamungsdyspnoe und einen Exophthalmus herbei, ganz wie bei der Maus, aber im Gegensatz zur letzteren keine Überempfindlichkeit gegen Berührungsreize und kein Betragen, das das Vorhandensein einer Hyperästhesie vermuten liess. Bei grossen Dosen erfolgte der Atemstillstand mit mehr oder weniger heftigen Konvulsionen. Die minimale Letaldosis betrug pro $10 \mathrm{~g}$ Körpergewicht etwa 2,0 ccm, war also viel grösser als die bei der Maus. Bei subletalen Dosen waren an der Injektionsstelle ebenfalls Induration und dann trockne Gangrän bemerkbar. Wiederholte tägliche Injektionen mit einer kleineren Dosis verursachten an den Extremitäten weder Rötung noch Anschwellung.

4) Kaninchen: Die subkutane Injektion von $200 \mathrm{ccm}$ des Extraktes pro $1 \mathrm{~kg}$ Körpergewicht hatte ausser einer vorübergehenden Atembeschleunigung, die vielleicht von der lokalen Reizung auf reflektorischem Wege ausgelöst wurde, keine andere in die Augen fallende akute Veränderung der Allgemeinerscheinungen zur Folge. An (ler Injektionsstelle entstand aber ebenfalls eine Induration, die sich später in eine trockene Nekrose verwandelte.

Bei täglicher Verabreichung von je $1 \mathrm{~g}$ getrocknetem Pilzpulver 
pro $1 \mathrm{~kg}$ Körpergewicht in den Magen setzten Appetitabnalme und Schwäche ein, die nach ungefähr 1 Woche bemerkbar und nach etwa 3 Wochen so auffallend wurden, dass das Tier stark abmagerte und herunterkam. Das Tier machte dann willkürlich keine lebhaften Bewegungen. Trotzdem versuchte es auf eine leise, aber plötzliche Berührung irgendeines Teils der Körperoberfläche mit der Hand schnell zu entlaufen, während es auf Schall jedoch gar nicht reagierte. Das Tier starb nach etwa 5 Wochen unter Marasmus. Während des ganzen Verlaufs der Vergiftung war aber an den Extremitäten weder Rötung noch Anschwellung bemerkbar.

5) Meerschweinchen: Die minimale Letaldosis des Extraktes betrug bei subkutaner Injektion bei diesem Tiere etwa $0,4 \mathrm{ccm}$ pro $10 \mathrm{~g}$ Körpergewicht. Bei dieser Dosis wurden die Atembewegungen anfänglich schneller, dann langsamer und mühsam und setzten später aus wie bei der Maus. Aber anders als bei der letzteren wurde das Meersch weinchen in einem Stadiun, in dem es auf der Seite lag, anfallsweise von heftigen klonischen und tonischen Krämpfen befallen. Indessen wurde die Atmung stark beeinträchtigt, so dass sie schliesslich unter Konvulsionen aufhörte. Nach dem Stillstand der Atmung schlug das Herz noch einige Zeit weiter. Bei der Sektion fand man in der Lunge immer eine auffallende Hyperämie.

Aus diesen Versuchen geht hervor, dass die minimale Letaldosis des Pilzextraktes pro $10 \mathrm{~g}$ Körpergewicht für den Frosch 0,4, für die Maus 0,3 und für die Ratte $2,0 \mathrm{~cm}$ beträgt. Das Kaninchen zeigt dagegen bei einer Dosis von $200 \mathrm{ccm}$ pro kg Körpergewicht gar keine Vergiftungserscheinungen. Die Empfindlichkeit der Tiere gegen das Gift ist also je nach ihrer Art ziemlich verschieden. Auch die Vergiftungssymptome weichen je nach den Tierarten beträchtlich von einander ab, beim Frosch bestehen sie in einer zentralen motorischen Lähmung, bei der Maus und Ratte ausserdem in einer Atemstörung und beim Meerschweinchen daneben in klonischen und tonischen Krämpfen. Bei der Maus tritt überdies noch ein Verhalten hinzu, das vielleicht auf Hyperästhesie beruht und das bei den anderen Tieren gänzlich fehlt. Die chronischen Vergiftungserscheinungen bei der Maus und dem Kaninchen sind Appetitlosigkeit und Kräfteschwund, die zum Tode der Tiere führen. Beim Kaninchen ist im Verlauf eine Reflexsteigerung bemerkbar, aber bei der Maus nicht. In der Entstehung der Induration und der trocknen Gangrän an der Injektionsstelle gibt es $\mathrm{z}$ wischen den beiden Tierarten keinen Unterschied. In allen Fällen konnten aber weder Rötung noch Anschwellung der Haut, die 
bei der Vergiftung des Menschen auftreten, beobachtet werden. Jedoch ist es höchst wahrscheinlich, dass die bei der Maus auftretende anscheinende Hyperästhesie den beim Menschen auftretenden Schmerzen im Wesen gleich ist.

\section{Wirkung auf die Atmung.}

Wie oben geschildert, führ't der Pilzextrakt bei der Maus, Ratte und dem Meerschweinchen gleichfalls eine Verlangsamungsdyspnoe herbei, während er daneben noch je nach den Tierarten verschjedene andere Vergiftungserscheinungen hervorruft. Um diese Veränderung der Atembewegungen ausfülırlich zu untersuchen, wurde der Extrakt einer mit Urethan narkotisierten, in der Rückenlage fixierten Ratte subkutan injiziert, bei der die Trachea mit einem Tambour und die Karotis mit einem $\mathrm{Hg}$-Manometer verbunden worden waren, damit die Atemberwegungen gleichzeitig mit dem Blutdruck registriert werden konnten. Bald nach der Darreichung einer letalen Dosis traten eine Beschleunigung und Vertiefung der Atembewegungen ein, die aber nach etwa 1 Minute zurückgingen. 1-2 Minuten danach begann dann die Atmung an Zahl und Tiefe wiederum zuzunehmen. Diese Zunahme, die viel stärker als die anfängliche, vorübergehende war, wurde immer deutlicher und erreichte nach etwa 3 Minuten ihr Maximum. Dieser Zustand hielt etwa 5 Minuten an und schlug dann ins Gegenteil um. Zur selben Zeit, als die Atemzüge sich an Zahl stark verringerten, fingen sie aber an, an Tiefe wieder zuzunehmen. Etwa 30 Minuten nach der Darreichung zeigte die Atmung eine beträchtliche Verlangsamung und Vertiefung, wobei das Tier unter Mitbewegung aller auxiliaren Atemmuskeln atmete. Die Tiefe begann danach wiederum abzunehmen, so dass dann die Atemverlangsamung und die Atemverflachung Hand in Hand Fortschritte machten. Aber in den meisten Fällen kam die Atmung, bevor ihre Tiefe stark beeinträchtigt wurde, plötzlich zum Stillstand, nur in seltenen Fällen wurde sie immer langsamer und seichter und hörte ganz allmählich auf. Der Blutdruck zeigte, wenn auch nicht immer, so doch häufig fast gleichzeitig mit der anfänglichen Atembeschleunigung eine leichte Steigerung, die aber mit dem Verschwinden der ersteren zurückging. Unablängig davon, ob diese Blutdruckveränderung erfolgte oder nicht, fing der Blutdruck etwa 2 Minuten nach der Injektion, als die zweite Atembeschleunigung einsetzte, zu steigen an, erreichte zur selben Zeit wie die Atembeschleunigung das Maximum, ging dann mit ihr zusam- 
men zurück und sank, als die Atmung sich stark verlangsamte und vertiefte, unter den normalen Wert. Als die Atmung nahezu oder völlig aufhörte, stieg der Blutdruck plötzlich über die normale Höhe, nahm aber bald danach ab und erreichte früher oder später den Nullwert. Wurde vorher die künstliche Respiration ausgeführt, so blieb der Blutdruck noch lange Zeit ungefähr in normaler Höhe stehen. Bei Versuchen an nicht narkotisierten Tieren liessen sich dieselbe Veränderungen der Atembewegungen und des Blutdruckes beobachten, obgleich die Kurven hierbei durch häufig einsetzende Körperbewegungen beeinträchtigt wurden. Ein Beispiel für diese Versuche ist in Fig. 1 wiedergegeben.
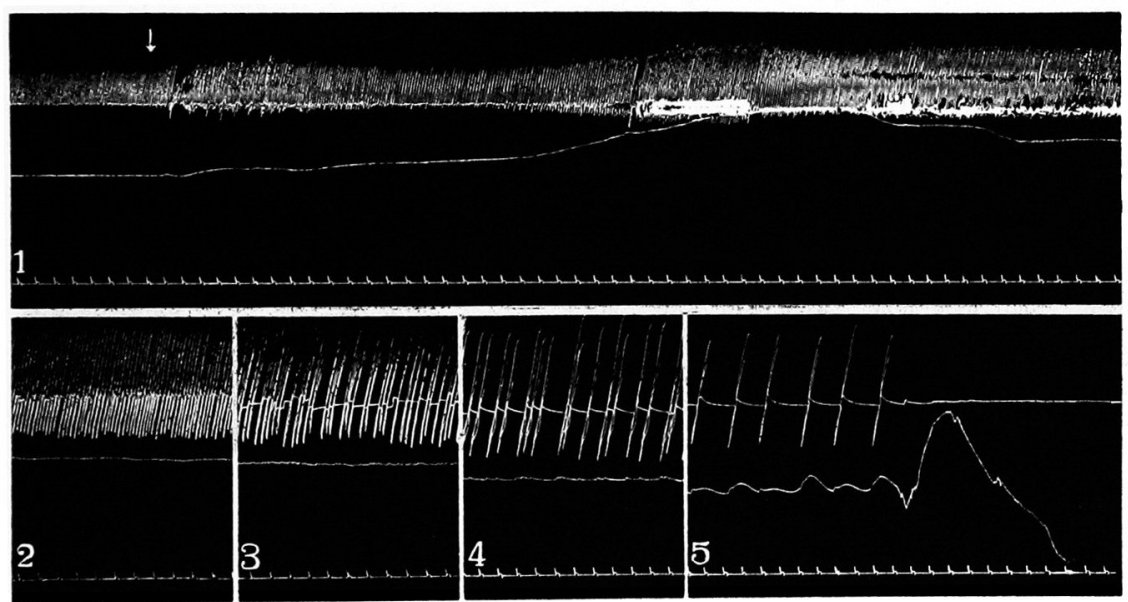

Fig. 1. Atembewegungen (oben) und Blutdruck (unten) einer urethanisierten Ratte von $230 \mathrm{~g}$ Körpergewicht. 1: Beginn des Versuches, 2: 15, 3: 30, 4:90, $5: 150$ Minuten später. Bei $\downarrow$ subkutane Injektion von $9,2 \mathrm{ccm}$ eines $50 \%$ igen Pilzextraktes. Zeitmarkierung in 5 Sekunden.

Wie aus diesen Versuchen an Ratten ersichtlich ist, werden durch subkutane Einverleibung des Pilzextraktes anfängliche, vorübergehende und darauffolgend langanhaltende Veränderungen der Atembewegungen und des Blutdruckes herbeigeführt. Die anfängliche Atembeschleunigung und Blutdrucksteigerung sind aber als eine mittelbare Folge der durch den Extrakt verursachten, lokalen, sensiblen Reizung zu betrachten, denn diese Veränderungen setzen so frühzeitig ein, dass eine resorptive Wirkung des Extraktes kaum erwartet werden kann. Da die darauffolgenden Respirationsveränderungen, d.h. die Beschleunigungs- und die Verlangsamungsdyspnoe, und die an- 
haltende Blutdrucksteigerung miteinander in keine wichtigen kausalen Beziehung stehen, so müssen die beiden durch die direkte Einwirkung des Extraktes auf die betreffenden Organe bedingt sein. Es wurden deshalb die folgenden Versuche angestellt, um die Veränderungen der Respiration und des Blutdruckes voneinander getrennt etwas näher zu studieren.

1) Veränderungen der Atembewegungen: Einer mit Urethan narkotisierten Ratte, deren beide Vagi am Halsteil durchschnitten worden waren, wurde eine tödliche Dosis des Extraktes subkutan gegeben. Bald nach der Injektion erfolgte ebenso wie beim Tier mit den intakten Vagi, eine vorübergehende Beschleunigung der Atembewegungen. Im Anschluss daran fingen die Atembewegungen an an Tiefe zuzunehmen wie beim Tier mit der intakten Vagusinnervation, während sie an Zahl dagegen keine Veränderung oder sogar eine geringe Verminderung erfuhren, wie Fig. 2 zeigt. Der weitere Verlauf deckte sich mit dem bei Tieren mit intakten Vagi.

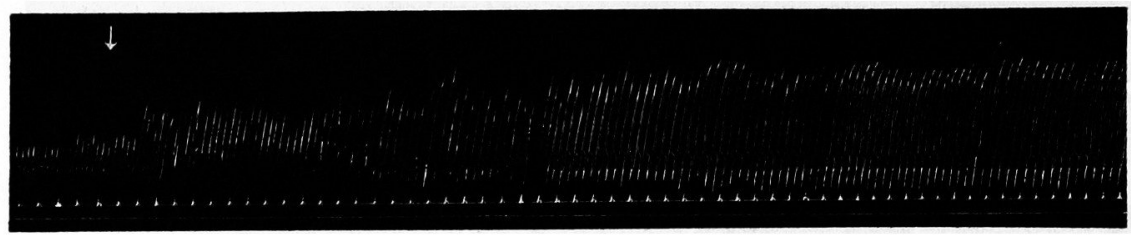

Fig. 2. Atembewegungen einer urethanisierten Ratte von $120 \mathrm{~g}$ Körpergewicht mit durchschnittenen Vagi. Bei $\downarrow$ subkutane Injektion von 4,8 ccm eines 50\%igen Pilzextraktes. Zeitmarkierung in 5 Sekunden.

Aus diesem Versuch ist zu ersehen, dass die Zunahme der Atemzahl, die beim Tier mit intakten Vagi auf die vorübergehen de Zunahme folgt, von der Wirkung des Extraktes aufs Vagusende herrührt, während die anderen Veränderungen der Atembewegungen mit dieser Wirkung nichts zu tun haben.

Bei einer mit dem Extrakt vergifteten Ratte bemerkte man in einem Stadium der hochgradigen Verlangsamungsdyspnoe, dass sich der Brustkorb bei der Inspiration erweiterte, aber der Bauch sich nicht mehr ausdehnte, sondern sich vielmehr zusammenzog. Um dieses Verhalten genauer zu untersuchen, wurde an der Brust- und Bauchhaut einer in der Rückenlage fixierten Ratte je ein Haken angebrecht, der mittels eines Fadens mit einem Schreibhebel verbunden wurde, damit die Bewegungen des Brustkorbs und des Bauches registriert werden konnten. Wie man in Fig. 3 sieht, trieben beide Teile vor 

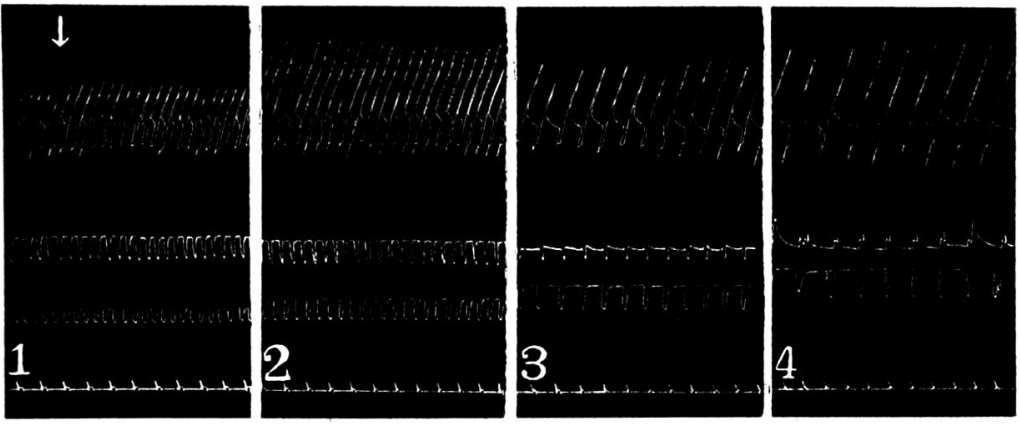

Fig. 3. Respiration (oben) sowie Bewegungen des Brustkolbs (unten) und des Bauches (mitte) einer urethanisierten Ratte von $140 \mathrm{~g}$ Körpergewicht. Bei $\downarrow$ subkutane Injektion von 5,6 ccm eines 50\%igen Extraktes. 1: Beginn des Versuchs und 2: 15, 3:30, 4:90 Minuten danach. Zeitmarkierung in 5 Sekunden.

und im Anfangsstadium der Vergiftung bei jeder Inspiration auf, während sie sich bei jeder Exspiration zusammenzogen. Nach dem Auftritt der starken Verlangsamungsdyspnoe zeigte der Bauch gerade das Gegenteil, er kontrahierte sich bei der Inspiration und erweiterte sich bei der Exspiration, der Brustkorb dagegen verhielt sich wie vorher.

Diese Versuche weisen darauf hin, dass im Stadium der hochgradigen Verlangsamungsdyspnoe das $Z$ werchfell an den Respirationsbewegungen nicht mehr aktiv beteiligt ist, sondern durch diese nur. passiv bewegt wird. Somit liegt die Vermutung sehr nahe, dass es sich dabei um eine Lähmung des Phrenicus handelt.

Um zu entscheiden, ob diese Auffassung zutreffend ist oder nicht, wurde bei einer urethanisierten Ratte, bei der der Nervus phrenicus, thoracicus longus und ischiadicus sowie das Zwerchfell blossgelegt worden waren, die Stärke, bei welcher eine elektrische tetanisierende Reizung der genannten Nerven oder des Zwerchfells eine bemerkbare Kontraktion der betreffenden Muskeln hervorzubringen imstande war, vor und nach subkutaner Verabfolgung des Pilzextraktes in einer tödlichen Dosis geprüft. Wie im nachstehenden Versuchsbeispiel erkennbar ist, zeigte die Reizstärke bei der Phrenicusreizung schon $30 \mathrm{Minu}-$ ten nach der Darreichung eine Zunahme, die mit der Zeit immer deutlicher wurde, so dass das Zwerchfell etwa 2 Stunden nach der Darreichung, als das Tier langsame, angestrengte Atemzüge machte, auf die Nervenreizung bei einem Rollenabstand von Null nicht mehr reagierte. Dagegen blieb die Reizstärke bei den anderen Nerven und dem Zwerchfell, bis der Atemstillstand erfolgte, fast unverändert. 
Versuchsbeispiel, Ratte von $170 \mathrm{~g}$ Körpergewicht. Urethannarkose.

\begin{tabular}{|c|c|c|c|c|c|}
\hline \multirow[b]{2}{*}{ Zeit } & \multicolumn{4}{|c|}{ Schwellenwert in cm R.A. bei Reizung des } & \multirow[b]{2}{*}{ Bemerkung } \\
\hline & $\begin{array}{c}\text { N. } \\
\text { thoracicus } \\
\text { longus }\end{array}$ & $\stackrel{N}{N}$ & $\begin{array}{c}\text { N. } \\
\text { phrenicus }\end{array}$ & $Z_{\text {werchfells }}$ & \\
\hline $6410^{\prime}$ & 26 & 25 & 25 & 17 & \\
\hline $20^{\prime}$ & 26 & 27 & 28 & 17 & \\
\hline $30 \%$ & 26 & 26 & 28 & $17 \mathrm{i}$ & \\
\hline $35^{\prime}$ & & & & & $\begin{array}{l}\text { Subkutane Injektion von } \\
6,8 \mathrm{ccm} \text { eines 50\%igen } \\
\text { Pilzextraktes. }\end{array}$ \\
\hline $7^{h}$ & 27 & 25 & 25 & 17 & Atmung beschleunigt. \\
\hline $30^{\prime}$ & 27 & 25 & 20 & 17 & $\begin{array}{l}\text { Atmung verlangsamt und } \\
\text { vertieft. }\end{array}$ \\
\hline $8^{\mathrm{b}}$ & 26 & 25 & 20 & 17 & $\begin{array}{l}\text { Atmung stark verlang- } \\
\text { samt. }\end{array}$ \\
\hline $9^{30^{\prime}}$ & $\begin{array}{l}26 \\
25\end{array}$ & $\begin{array}{l}24 \\
24\end{array}$ & $\begin{array}{c}12 \\
\text { bei o keine }\end{array}$ & 16 & Atmung mühsom. \\
\hline $\begin{array}{l}30^{\prime} \\
40^{\prime}\end{array}$ & 25 & 24 & Reaktion. & 18 & Atmung steht still. \\
\hline
\end{tabular}

Dieser Versuch beweist deutlich, dass der Pilzextrakt vor allem auf die Phrenicusenden wirkt, diese ausser Funktion setzt und dadurch eine angestrengte Kostalatmung verursacht. Es ist auch begreiflich, dass der Extrakt auf das Respirationszentrum lähmend einwirkt und dadurch eine Verlangsamung und schliesslich den Stillstand der Atembewegungen herbeiführt.

2) Veränderung des Blutdruckes: Wie oben bemerkt, ruft der Pilzextrakt bei Ratten in einer tödlichen Dosis deutliche Veränderungen des Blutdruckes hervor, die in einer anfänglichen, reflektorischen, schnellvorübergehenden und in einer darauffolgenden, langanhaltenden Steigerung sowie in einer später eintretenden Herabsetzung bestehen. Bei Versuchen mit einer Dosis von $4 \mathrm{ccm}$ eines $50 \%$ igen Extraktes pro $100 \mathrm{~g}$ Körpergewicht trat die ebengenannte anhaltende Steigerung des Blutdruckes mit oder ohne vorangehenden kurzen Anstieg ein. Die erstere machte dann einer Herabsetzung Platz, wobei der Blutdruck allmählich auf die normale Höhe herabsank, aber nie unter diesen Wert ging. Um die Ursache dieser Blutdruckveränderung erklären zu können, wurden Versuche am Herzen und an den Gefässen durchgeführt.

3) Herz: Bei einer mit Urethan narkotisierten Ratte wurde der Karotisdruck mittels eines $\mathrm{Hg}$-Manometers registriert, und unter Ausführung künstlicher Atmung der Brustkorb geöffnet, um das Herz blosszulegen, dessen Kontraktionen dann nach der Methode von Tak a- 

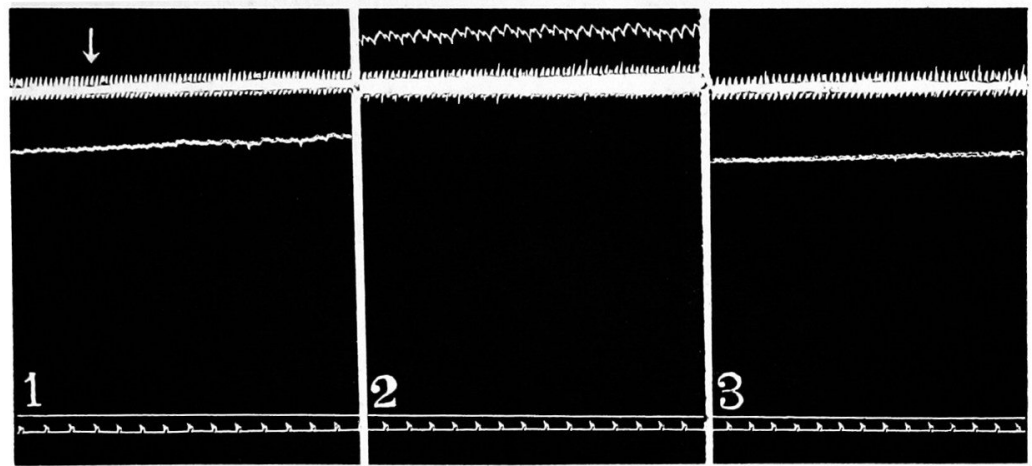

Fig. 4. Ventrikelkontraktionen (oben) und Karotisdruck (unten) einer urethanisierten Ratte von $200 \mathrm{~g}$ Körpergewicht bei künstlicher Atmung. 1: Beginn des Versuchs, 2: 20 und 3: 50 Minuten später. Bei $\downarrow$ subkutane Injektion von $4 \mathrm{ccm}$ eines 50\%igen Extraktes. Zeitmarkierung in 5 Sekunden.

hashi und anderen ${ }^{3)}$ aufgezeichnet wurden. Nach subkutaner Injektion von $2 \mathrm{ccm}$ des Extraktes pro $100 \mathrm{~g}$ erfolgte eine Blutdrucksteigerung, die aber weniger deutlich und anhaltend war als die bei den vorigen Versuchen. Die Ventrikelkontraktionen fingen mit dem Beginn der Blutdrucksteigerung an, an Umfang zuzunehmen, zeigten bei der höchsten Blutdrucksteigerung die maximale Vergrösserung und nahmen mit der Blutdruckherabsetzung wieder ab, so dass die Kontraktionen und der Blutdruck früher oder später zu dem ursprünglichen Zustand zurückkehrten, wie Fig. 4 zeigt. Dabei erfuhren die Ventrikelkontraktionen an Zahl fast keine Veränderung.

Hiernach ist die anhaltende Blutdrucksteigerung als Folge der Vermehrung der Herzarbeitsleistung zu betrachten, die die Vergrösserung des einzelnen Schlagvolumens betrifft, wenn auch noch nicht klar isi, ob es sich dabei noch um ein anderen Faktor handelt.

Da Versuche an Fröschen, bei denen das Herz blossgelegt und der Pilzextrakt in den Oberschenkellymphsack gegeben wurde, ergaben, dass sich das Herz gegen den Extrakt ganz wie das der Ratten verhielt, wurde die Herzwirkung des Extraktes am isolierten Froschherzen untersucht, um dadurch die erwähnten Veränderungen der Herzschläge bei der Ratte mittelbar erklären zu können. Das herausgeschnittene Herz eines Frosches wurde mit Hilfe eines künstlichen Zirkulationsapparates mit $5 \mathrm{ccm}$ Ringerlösung ernährt. Nach Zusatz von einem Tropfen eines 10\% igen Extraktes zur Nährfliissigkeit trat

3) Takahashi u. a., Tohoku Journ. of Exp. Med. 1936, 30, 85. 
in den Ventrikelkontraktionen eine deutliche Zunahme des Umfangs ein, die die Verstärkung der Systole und das Vollkommenerwerden der Diastole betraf, und nach langer Dauer allmählich zurückging. Dabei zeigte die Frequenz in den meisten Fällen keine Veränderung, erfuhr aber selten eine leichte Zunahme.

Bei der Vermehrung der Dosis folgte auf die eben erwähnte Veränderung eine Abnahme der Schlagzahl der Kammer und der Vorhöfe. Die Abnahme ging schneller bei der ersteren als bei den letzteren vor sich, so dass zwischen den Kammer- und den Vorhofkontraktionen eine Dissoziation auftrat und die Kammer schliesslich, während die Vorhöfe langsam, aber doch noch regelmässig fortschlugen, in der Diastole stillstand. Dabei antwortete die Kammer auf mechanische Reizung prompt mit einer starken Kontraktion. Später kamen auch die Vorhöfe zum Stillstand bevor die Sinuspulsationen aufhörten.

Daraus geht hervor, dass der Pilzextrakt durch seine unmittelbare Wirkung auf das Froschherz eine Amplitudenvergrösserung verursacht. In grossen Dosen lähmt er das Reizleitungssystem und verursacht dadurch eine Abnabme der Schlagzahl und schliesslich den Stillstand des Herzens. Die Herzwirkung des Extraktes auf das Rattenherz könnte vielleicht analog erklärt werden.

4) Gefässe: Da es schwierig war, bei Ratten den Gefässzustand verschiedener Organe onkometrisch zu untersuchen, wurden Durchströmungsversuche mit einer gummihaltigen Ringerlösung an abgeschnittenen Hinterschenkeln von Ratten ausgeführt, um die Wirkung des Extraktes auf die Gefässe klar zu machen. Die niedrigste Konzentration des Extraktes, die eine bemerkbare Veränderung in der Ausflussflüssigkeit herbeizuführen vermochte, betrug bei Einspritzung ins Einflussrohr in einer Menge von $0,1 \mathrm{ccm} 0,01 \%$. Die dadurch verursachte Veränderung war immer eine Verminderung, die mit der Steigerung der Konzentration deutlicher wurde. Bei einem $0,1 \%$ igen Extrakt z. B. setzte die Verminderung bald nach der Injektion ein und gelangte in 3-4 Minuten zum höchsten Wert, wobei die Menge der Ausflussflüssigkeit etwa $\frac{1}{3}$ der anfänglichen betrug. Die Ausflussmenge nahm danach langsam mehr und mehr zu, erreichte aber niemals die anfängliche. Somit ist es sicher, dass der Extrakt bei Ratten durch seinen peripheren Angriff eine Gefässverengerung bewirkt.

Das Rattenschenkelpräparat erwies sich wegen rascher Ödembildung als unzweckmässig, in die Gefässwirkung näher einzugehen. Deshalb wurde die weitere Untersuchung an Froschschenkelpräparaten nach dem Lae wen-Trendelen burgschen Verfahren vorgenom- 
men. In diesem Fall erfolgte bald nach der Einführung von $0,1 \mathrm{ccm}$ eines 0,1\% igen Extraktes ins Einflusrohr eine Verminderung der Ausflussflüssigkeit; die nach etwa 3 Minuten den höchsten Wert zeigte, und dann in eine Vermehrung umschlug, wie beim vorigen Versuch. Die Ausflussflüssigkeit erreichte aber 5-10 Minuten später wieder den alten Zustand.

Bei einem Froschschenkelpräparat, bei dem $0,2 \mathrm{ccm}$ von einer 0,5\% igen Ergotoxinlösung ins Einflussrohr eingespritzt worden war, wodurch die Injektion von 0,1 $\mathrm{ccm}$ einer Adrenalinlösung von $1: 10,000,000$ unwirksam gemacht wurde, war die Injektion von 0,1 ccm eines 0,1\%igen Extraktes, die sonst eine deutliche Verminderung der Ausflussflüssigkeit hervorzurufen imstande war, ebenfalls unfähig eine solche herbeizuführen. Etwa 1 Stunde nach der Ergotoxindarreichung, wo die gleiche Menge von Adrenalin wieder eine Verminderung der ausfliessenden Flüssigkeit bewirken konnte, hatte der Pilzextrakt in der gleichen Menge ebenfalls eine Verzögerung des Flüssigkeitsausflusses zur Folge. Daraus folgt, dass der Pilzextrakt wie das Adrenalin die sympathischen Nervenenden erregt und eine Gefüssverengerung verursacht.

Langsame Einführung von $1 \mathrm{ccm}$ eines 1\%igen Extraktes ins Einflussrohr eines Froschschenkelpräparates verursachte eine vollständige Sistierung des Ausflusses, die aber nach etwa 15 Minuten fast gänzlich verschwand. In diesem Stadium bewirkte die Darreichung von 0,2 ccm der Adrenalinlösung gar keine Abnahme der Ausflussflüssigkeit, während die von $0,2 \mathrm{ccm}$ einer $0,5 \%$ igen Chlorbariumlösung eine entscheidende Verminderung derselben hervorbrachte. Sonach ist es höchst wahrscheinlich, dass der Pilzextrakt in grossen Dosen ebenso wie das Ergotoxin die sympathischen Nervenenden der Gefässe lähmt.

III. Wirkung auf die Glattmuskelorgane.

Das Ergebnis, dass der Pilzextrakt auf die sympathischen Nervenenden der Gefässe in kleinen Mengen erregend, in grossen dagegen lähmend einwirkt, bringt auf die Vermutung, dass es auch auf die anderen Glattmuskelorgane dieselbe Wirkung auszuüben mag. Um diese Frage klarzulegen, wurde eine Untersuchung am herausgeschnittenen Darm und Uterus des Kaninchens ausgeführt.

1) Darm: Ein herausgeschnittenes Dünndarmstück wurde in einem $100 \mathrm{ccm}$ Tyrodelösung enthaltenden Bad suspendiert, um 
seine Bewegung zu registrieren. Die erkennbare Veränderung des Darmzustandes trat erst bei Zusatz von $0,1 \mathrm{ccm}$ eines $10 \%$ igen Extraktes ins Bad ein und bestand in einer vorübergehenden Vergrösserung des Bewegungsumfangs. Die Vergrösserung wurde mit der Vermehrung des Extraktes auffallender und hielt länger an. Aber bei Zusatz von $1 \mathrm{ccm}$ wurde die Amplitude infolge einer Tonussteigerung beeinträchtigt, so dass die erstere unverändert blieb oder vielmehr eine Verkleinerung erlitt. Diese Tonussteigerung wurde durch Zusatz von 0,1 ccm einer $0,1 \%$ igen Atropinlösung prompt und vollständig beseitigt, wie dies Fig. 5 anschaulich macht.

Wenn ein Darmstück vorher durch $\mathrm{Be}$ handlung mit der gleichen Menge Atropin gegen Zusatz von 0,2 ccm einer $1 \%$ igen Pilocarpinlösung unempfindlich gemacht worden war, so folgte auf die Darreichung von 1$3 \mathrm{ccm}$ des Extraktes gar keine Steigerung des Tonus, ja sogar gelegentlich eine Herabsetzung desselben. Fig. 6 lässt sich dies deutlich erkennen.

Aus diesen Versu-

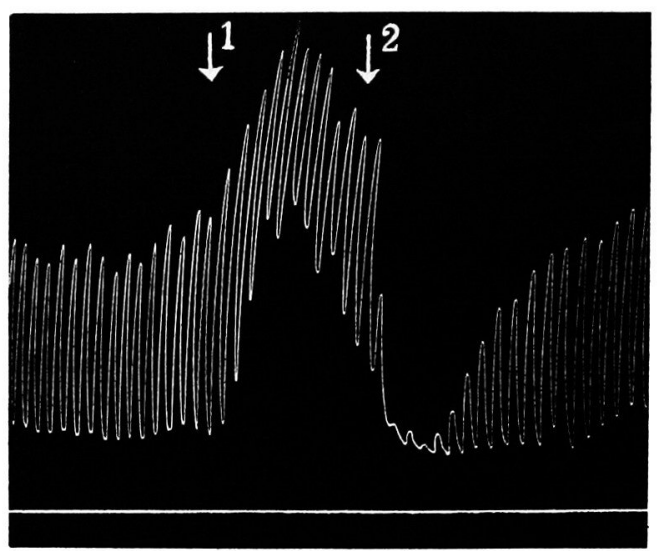

Fig. 5. Herausgeschnittener Kaninchendarm in einem Bad von $100 \mathrm{ccm}$ Tyrodelösung. Bei $\downarrow^{1} \mathrm{Zu}-$ satz von $1 \mathrm{ccm}$ eines $10 \%$ igen Pilzextraktes, bei $\downarrow^{2}$ Zusatz von $0,1 \mathrm{ccm}$ einer $0,1 \%$ igen Atropinlösung.

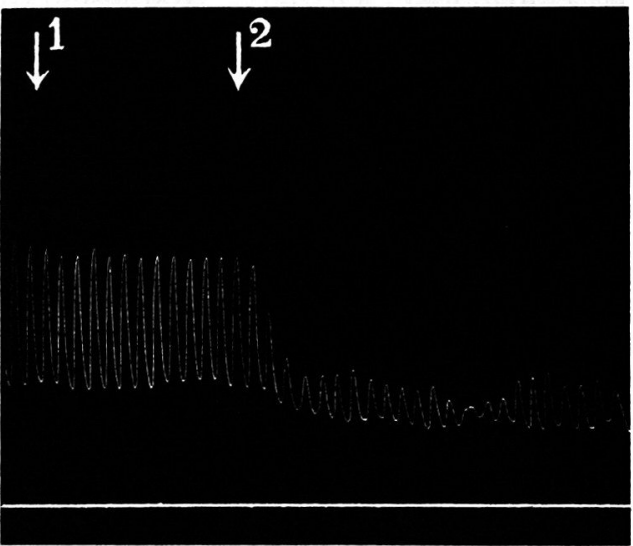

Fig. 6. Herausgeschnittener Kaninchendarm in einem Bad von $100 \mathrm{ccm}$ Tyrodelösung, der vorher mit $0,1 \mathrm{ccm}$ einer $0,1 \%$ igen A tropinlösung vergiftet worden ist. Bei $\downarrow^{1}$ Zusatz von $0,2 \mathrm{ccm}$ einer $1 \%$ igen Pilocarpinlösung, bei $\downarrow^{2}$ Zusatz von $2 \mathrm{ccm}$ eines 10\%igen Pilzextraktes. 
chen erklärt sich, dass der Pilzextrakt auf die Vagusenden des Darmes erregend einwirkt und dadurch eine Bewegungsförderung sowie eine Tonussteigerung desselben hervorruft. Diese Tatsache scheint die Erwartung nicht zu erfüllen, dass der Extrakt auch die sympathischen Nervenenden des Darmes anzugreifen vermag. Wenn man aber darauf Rücksicht nimmt, dass der durch den Extrakt gesteigerte Tonus durch Atropin bis weit unter den früheren herabgesetzt wird und dass der Extrakt den Tonus des atropinisierten Darms senkt, so liegt doch die Vermutung nahe, dass der Extrakt auch die sympathischen Nervenenden erregt. Wahrscheinlich ist hierbei die Erregung der Vagusenden der der Symphathicusenden an Stärke uberlegen, so dass nur eine Tonussteigerung und eine Bewegungsförderung erfolgen.

2) Uterus: Ein herausgeschnittenes Uterusstück eines erwachsenen nicht trächtigen Kaninchens wurde wie bei den vorigen Versuchen vorbereitet. Auf Zusatz von $0,1 \mathrm{ccm}$ eines $10 \%$ igen Extraktes ins Bad folgte eine leichte, doch unverkennbare Steigerung des Tonus, die allmählich verschwand. Die Tonussteigerung nahm mit Vermehrung der zugesetzten Menge an Stärke sowie an Dauer immer zu. Und bei einer $\mathrm{Zu}-$ satzdosis von $2 \mathrm{ccm}$ war sie sehr auffallend, so dass die Bewegungen

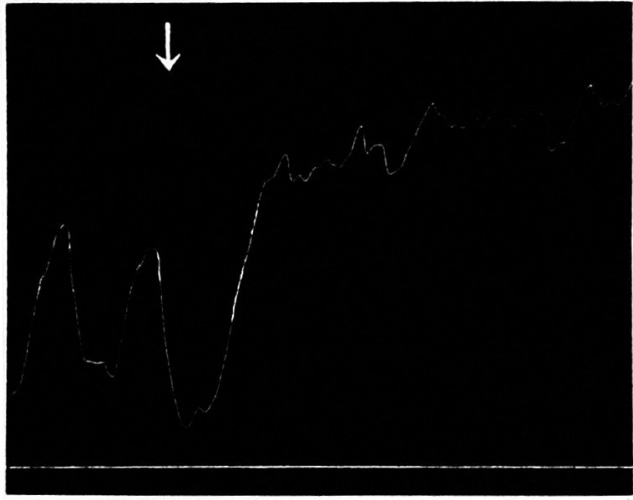

Fig. 7. Herausgeschnittener Kaninchenuterus in einem Bad von $100 \mathrm{ccm}$ Tyrodelösung. Bei $\downarrow$ Zusatz von $2 \mathrm{ccm}$ eines 10\%igen Extraktes. stark oder vollständig gehemmt wurden. Fig. 7 zeigt ein Beispiel für diese Versuche.

Als der Tonus eines Uterusstückes durch Einwirkung von $2 \mathrm{ccm}$ des Extraktes gesteigert worden war, wurde er durch Zusatz von $0,1 \mathrm{mg}$ Atropin erheblich, aber nicht vollständig, und erst durch den darauffolgenden Zusatz von $0,2 \mathrm{mg}$ Ergotoxin ungefähr bis auf den normalen Wert heruntergebracht. Bei einem Uterusstück, das vorher mit $10 \mathrm{mg}$ Atropin oder mit 0,2 $\mathrm{mg}$ Ergotoxin vergiftet worden war, war der Zusatz von 1,0 ccm Extrakt noch imstande, eine deut- 

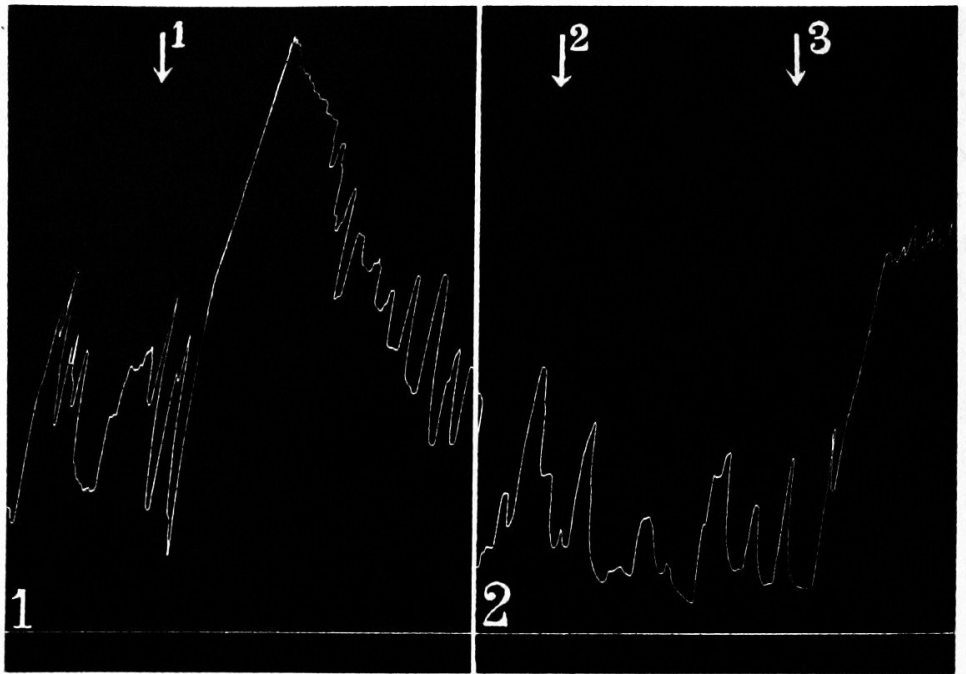

Fig. 8. Herausgeschnittener Kaninchenuterus in einem Bad von $100 \mathrm{ccm}$ Tyrodelösung. Bei $\downarrow^{1}$ Zusatz von $2 \mathrm{~cm}$ eines $10 \%$ igen Pilzextraktes, bei $\downarrow^{2}$ Zusatz von $1 \mathrm{ccm}$ einer Adrenalinlösung von $1: 100000$, und bei $\downarrow^{3}$ Zusatz von $0,2 \mathrm{ccm}$ einer 1\%igen Pilocarpinlösung ins Bad.

liche Tonussteigerung zu erzeugen. Wenn ein Uterusstück vorläufig mit $10 \mathrm{mg}$ Atropin und 0,2 mg Ergotoxin behandelt wurde, so vermochte die gleiche Menge des Extraktes nicht mehr eine Tonussteigerung zu verursachen.

Aus diesen Versuchen gèht eindeutig hervor, dass der Pilzextrakt beim Kaninchenuterus sowohl auf die sympathischen als auch auf die parasympathischen Nervenenden eine erregende Wirkung ausübt und dadurch eine Tonussteigerung herbeiführt. Dieses Ergebnis dient vielleicht dazu die Auffassung zu unterstützen, dass der Extrakt auch beim Darm neben den parasympathischen noch die sympathischen Nervenenden in Erregung versetzt.

Wenn man den Extrakt in einer grossen Menge wie $2 \mathrm{ccm}$ längere Zeit auf ein Uterusstück einwirken liess, so sah man, dass der Tonus nach einer langandauernden Steigerung allmählich herabgesetzt wurde und schliesslich an den ursprünglichen Wert herankam. In diesem Stadium bewirkte der Zusatz von Adrenalin keinen Tonusanstieg, der aber durch Zusatz von Pilocarpin erzeugt werden konnte, wie Fig. 8 zeigt.

Das Pilzextrakt scheint also in grossen Dosen die sympathischen. Nervenenden, aber nicht die parasympathischen zu lähmen. 
III. Sensibilitätsstörung bei der Maus.

Wie oben erwähnt, bewirkt der Pilzextrakt bei der Maus eigentümliche Körperbewegungen, die vielleicht durch Parästhesie oder Hyperästhesie bedingt sind. Um auf die Entstehung dieser Erscheinungen etwas näher einzugehen, wurden die folgenden Versuche angestellt.

Wurde einer Maus, deren Grosshirn 24 Stunden vorher entfernt worden war, eine tödliche Dosis des Extraktes subkutan eingespritzt, so traten neben der Verlangsamungsdyspnoe eigentümliche Körperbewegungen ein, die in Lecken des Bauches und der Extremitäten, Aneinanderreiben der Vorderfüsse, Kratzen der Flanken mit den Hinterfüssen und Umherlaufen auf den Fusspitzen bestanden. Der Tod erfolgte unter asphyktischen Konvulsionen. Diese Vergiftungserscheinungen stimmen mit denen bei Tieren mit Grosshirn vollständig überein, so dass man annehmen muss, dass die in Frage kommenden eigentümlichen Körperbewegungen wie auch die anderen Vergiftungserscheinungen in der Entstehnung mit dem Grosshirn in keinem wichtigen Zusammenhang stehen.

Gab man die gleiche Menge des Extraktes einer Maus, deren Rückenmark 3 Stunden vorher zwischen dem 7. Hals- und dem 1. Brustwirbel durchschnitten worden war, so bemerkte man ebenfalls die eigentümlichen Körperbewegungen, wenn sie auch an Intensität den im vorigen Versuch geschilderten nachstanden. In einem Stadium, wo das Tier auf der Seite lag und seine Extremitäten unabsichtlich bewegte, veranlasste eine kurze leise Berührung der Extremitäten, die sonst keine Abwehrbewegungen auszulösen vermochte, heftige Bewegungen nicht nur der betreffenden Extremität, sondern auch der anderen Extremitäten, die so aussahen, als ob das Tier irgendetwas von der betreffenden Stelle entfernen wollte. Somit ist anzunehmen, dass die eigentümlichen Körperbewegungen durch die Wirkung irgendwo auf dem durch das Rückenmark vermittelten Reflexwege zustande kommen.

Spritzte man einer vergifteten Maus im Stadium, in dem die eigentümlichen Bewegungen stark ausgeprägt waren, $0,2 \mathrm{ccm}$ einer $0,5 \%$ igen Cocainlösung um den Ischiadicus herum in den einen Gesässteil ein, so bemerkte man, dass die anscheinenden Abwehrbewegungen der betreffenden Extremität verschwanden, und das Glied imstande war, die normalen Bewegungen auszuführen. Dieses Ergebnis deutet darauf hin, dass die in Frage kommenden Vergifrungserschei- 
nungen nicht von der Wirkung auf die motorischen Nerven oder das Rückenmark, sondern von derjeniger auf die sensiblen Nerven abhängig sind.

Bei einer in der Bauchlage gefesselten Maus wurden die beiden $\mathrm{Nn}$. ischiadici an den Oberschenkel blossgelegt, von der Umgebung vorsichtig abgetrennt und durch Verschliessen der Wunden vor dem Trocknen geschützt. Nach etwa 5 Stunden wurden die beiden Nerven wieder freigelegt, von ihnen wurde der eine dann mit einem Stück Watte, das mit einem 10\%igen Extrakt getränkt wurde, und der andere mit einem solchen, das mit Ringerlösung befeuchtet wurde, bedecket. Dann wurden die Reflexbewegungen der hinteren Extremitäten, die durch mechanische Reizung der Fusspitze von einer bestimmten Stärke ausgelöst wurden, von Zeit zu Zeit untersucht. Hierbei konnte aber gar kein Unterschied in den Reflexbewegungen zwischen beiden Nerven innerhalb einer Stunde konstatiert werden. Die sensiblen Nervenfasern scheinen also vom Pilzextrakt nicht beeinflusst zu werden. Infolgedessen muss die eigentümliche Vergiftungserscheinung als Folge einer Erregbarkeitssteigerung der sensiblen Nervenenden aufgefasst werden.

\section{Zusammenfassung.}

Ein wässeriger Extrakt des Pilzes Clitocybe acromelalga, Ichimura, dessen Genuss bei uns bisweilen mehr oder weniger schwere Vergiftungen hervorruft, verursacht bei Tieren ebenfalls Vergiftungserscheinungen, die je nach den Tierarten sehr verschieden sind und von denen bei Menschen vor allem darin abweichen, dass bei keinem Tier zu Gangräne führende Rötungen und Schwellungen der Extremitäten eintreten.

Die Vergiftungserscheinungen bestehen beim Frosch hauptsächlich in einer Lähmung der willkürlichen und der Reflex- und Atembewegungen von zentraler Natur, bei der Maus, Ratte sowie dem Meerschweinchen daneben auch in einer den Atemstillstand herbeiführenden Verlangsamungdyspnoe, die nach der Untersuchung an der Ratte auf einer Lähmung des Phrenicus und des Atemzentrums beruht. Das letztgenannte Tier wird auch von anfallsweise auftretenden klonischen und tonischen Krämpfen befallen, die bei den anderen Tieren gänzlich fehlen. Von den untersuchten Tieren ist nur bei der Maus eine beträchtliche Erregbarkeitssteigerung der sensiblen Nervenenden zu beobachten, die ihrer Natur nach vielleicht dem bei der 
Vergiftung des Menschen eintretenden heftigen Schmerzen gleich zu sein scheint.

Es bewirkt wahrscheinlich infolge einer Erregung der sympathischen Nervenenden eine Vergrösserung des Schlagumfangs des Herzens und eine Verengung der Gefässe und führt dadurch eine Blutdrucksteigerung herbei.

Beim Uterus erregt es sowohl die sympathischen als auch die parasympatischen Nervenenden, während es beim Darm vorzüglich die letzteren in Erregung versetzt. Infolgedessen erfahren die beiden Organe eine Tonussteigerung und Bewegungsförderung.

Die mannigfaltigen Wirkungen des Extraktes beruhen möglicherweise darauf, dass in ihm verschiedene wirksame Substanzen enthalten sind, worüber später berichtet werden soll. 